Patricia Whitelock and Russell Cannon, eds.

\title{
HST Color-Magnitude Diagrams of Globular Clusters in NGC 185 and NGC 205
}

\author{
Doug Geisler and Taft Armandroff \\ Kitt Peak National Observatory, NOAO, Tucson AZ, 85719 USA
}

Gary Da Costa

Australian National University, Canberra, Australia

Myung Gyoon Lee

Seoul National University, Seoul, South Korea

Ata Sarajedini

San Francisco State University, San Francisco, CA 94132 USA

\begin{abstract}
.
We present a progress report on our ongoing HST WFPC2 study of globular clusters in NGC 185 and NGC 205. Most of the cluster candidates studied previously only from the ground are indeed globular clusters; however, several candidates turn out to be either foreground stars or background galaxies. In addition, we have discovered one new, previously unsuspected cluster. A complete knowledge of the globular cluster systems in even these nearby galaxies requires high spatial resolution data. We then derive preliminary $I,(V-I)$ color-magnitude diagrams for some of the clusters, as well as for their surrounding fields. The clusters show the blue horizontal branches expected for the low metallicities we derive, which are in agreement with those derived from ground-based integrated spectra. The fields appear generally more metal-rich than indicated by previous ground-based studies. The field blue horizontal branch in NGC 185 is only a very minor component, while that in NGC 205 is even smaller. After all of our observations have been acquired, these data will allow a very accurate knowledge of the individual and composite properties of the globular cluster systems of these 2 galaxies, as well as those of their field stars.
\end{abstract}

\section{Introduction}

The Local Group dwarf elliptical galaxies NGC 185 and NGC 205 each possesses its own small coterie of globular clusters (GCs), which can be as useful as those in our own Galaxy for studying early galaxy formation history, but whose horizontal branches are well beyond the reach of ground-based telescopes. Recent work 
by Durrell et al. (1996) and Miller et al. (1999), e.g., indicate the importance of the GCs in dwarf ellipticals for studying galaxy formation.

The GCs of NGC 185 were first discovered and initially investigated by Baade (1944), Hodge (1974) and Ford et al. (1977). The latter study summed up our knowledge of the census of clusters in this galaxy: they listed 6 probable and 2 possible GCs. A number of studies have investigated the GCs associated with NGC 205, mostly during searches for GCs around the nearby Andromeda galaxy. Compiling the work of Hubble (1932), van den Bergh (1969), Sargent et al. (1977) and Battistini et al. (1987), we find a total of 8 "probable" GCs and 10 additional candidates. The task of separating out a given cluster's association with either NGC 205 or M31 is problematical, though radial velocity can provide some discrimination.

We were granted 40 orbits of HST time as part of cycle 6 in order to obtain WFPC2 $V, I$ photometry for 5 GCs in each of these two galaxies. Each GC is observed for 1 orbit with the F555W filter and 3 orbits with the F814W, with the cluster centered on the PC, and the instrument oriented to include as many other candidates as possible. At this point, we have obtained data for half of our clusters. We present here a progress report on these data and some preliminary results.

\section{Cluster Census}

It is of some interest to examine the census of clusters in each galaxy. The high spatial resolution of the WFPC2 allows us to not only obtain very deep photometry for these objects but to also simply verify their cluster nature. Previous studies of these objects were all from the ground and were generally obtained with photographic plates with coarse plate scale, on which even large, bright clusters are often not easily resolved.

We have in hand data for 7 of Ford et al.'s (objects designated FJJ) 8 "GCs" in NGC 185 (the 8th object, FJJVIII, 1 of the 2 "possible" GCs, will not be observed). We find that 5 of Ford et al.'s "probable" GCs are indeed clusters. The other "possible" GC is also a cluster. Interestingly, the 6th "probable" GC, FJJVI, is actually an elliptical galaxy! In NGC 205, we have now observed 7 objects. Observations of 6 more objects should be obtained soon, while 5 more objects will not be observed. All 3 Hubble (1932 - designated H) objects observed are GCs. One of the Battistini et al. objects (a class "B" object, indicating it is very likely to be a GC) is a GC while 2 Battistini et al. class $\mathrm{B}$ objects are in fact stars. Another Battistini class $\mathrm{C}$ object is very faint and buried in a very crowded field, and without detailed photometry it is difficult to determine whether it is a true cluster or perhaps a random superposition of field stars. Finally, most interestingly, we have discovered what is almost certainly a new GC in this galaxy, previously unknown. It lies very close to the nucleus and is very compact, so that previous groundbased studies were unable to unambiguously determine its nature. It is also very blue and is likely a young cluster, similar to the one discussed by Da Costa \& Mould (1988).

Thus, of the total number of 7 cluster candidates in NGC 185 observed by us, 6 turn out to be true clusters, while in NGC 205 4-5 of our 7 observed candidates are GCs, and we have discovered a new cluster. Clearly, previous 


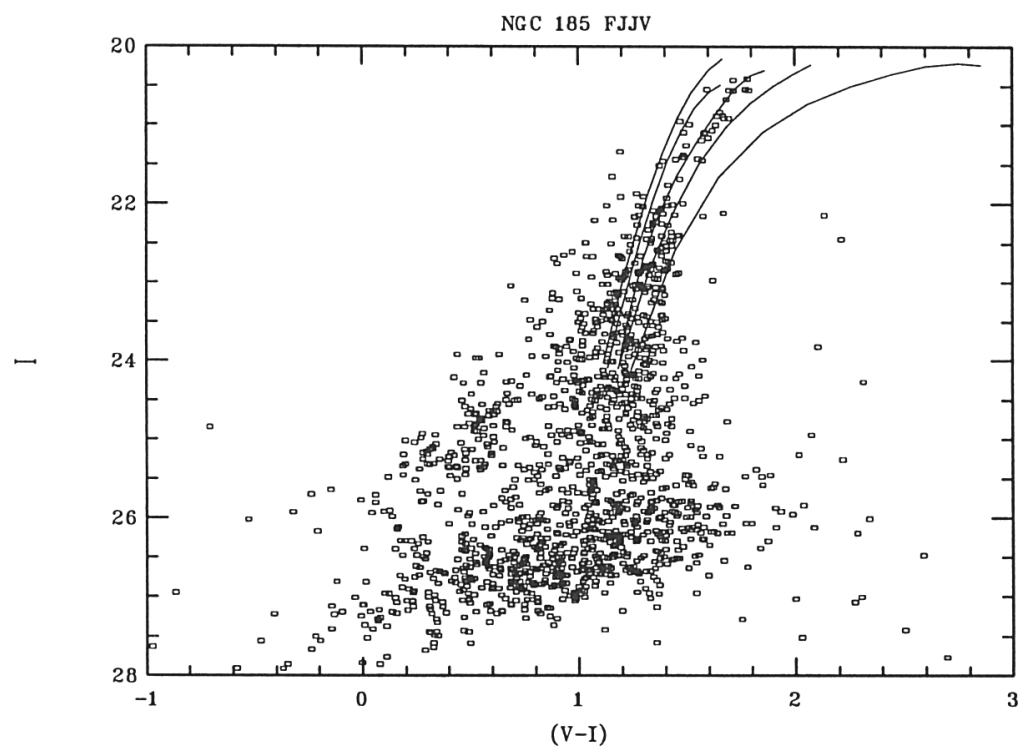

Figure 1. Preliminary $I$ vs. $(V-I)$ CMD for cluster FJJV in NGC 185. Only objects within $4.6^{\prime \prime}$ of the cluster center are plotted. We have overplotted Da Costa \& Armandroff (1990) standard giant branches, after correcting them for $E(V-I)=0.25$ and $(m-M)_{V}=24.5$.

groundbased studies using coarse-scale telescopes and mediocre seeing are not optimum for unambiguously identifying GCs in even these nearby galaxies. A similar conclusion has been reached by Geisler et al. (1999) in their modern survey of the M31 GC system. Observations with high spatial resolution, using either HST or groundbased CCDs with small pixels during times of excellent seeing, are required for definitive studies of these GCs.

\section{Preliminary Cluster Results}

We have currently produced preliminary results for 3 GCs in NGC 185 and 1 in NGC 205. Reductions have basically followed the prescription used by the HST $H_{0}$ Key Project team, which uses their own psf's and the multiple-image psffitting capabilities of ALLFRAME (Stetson 1994) to obtain photometry. We stress that the photometry presented herein is preliminary; in particular, we have not yet obtained aperture corrections. These should have little effect on the derived colors but may change the magnitudes by $\sim 0.05$ mag.

Figure 1 presents our CMD for FJJV in NGC 185. The photometry is challenging: this is a compact cluster buried in an already crowded background field. Nonetheless, given the excellent resolution of the PC and limiting the cluster sample to a narrow spatial range, the main features of the cluster are visible. The blue horizontal branch is clearly seen between $(V-I) \sim 0.2-0.8$ 


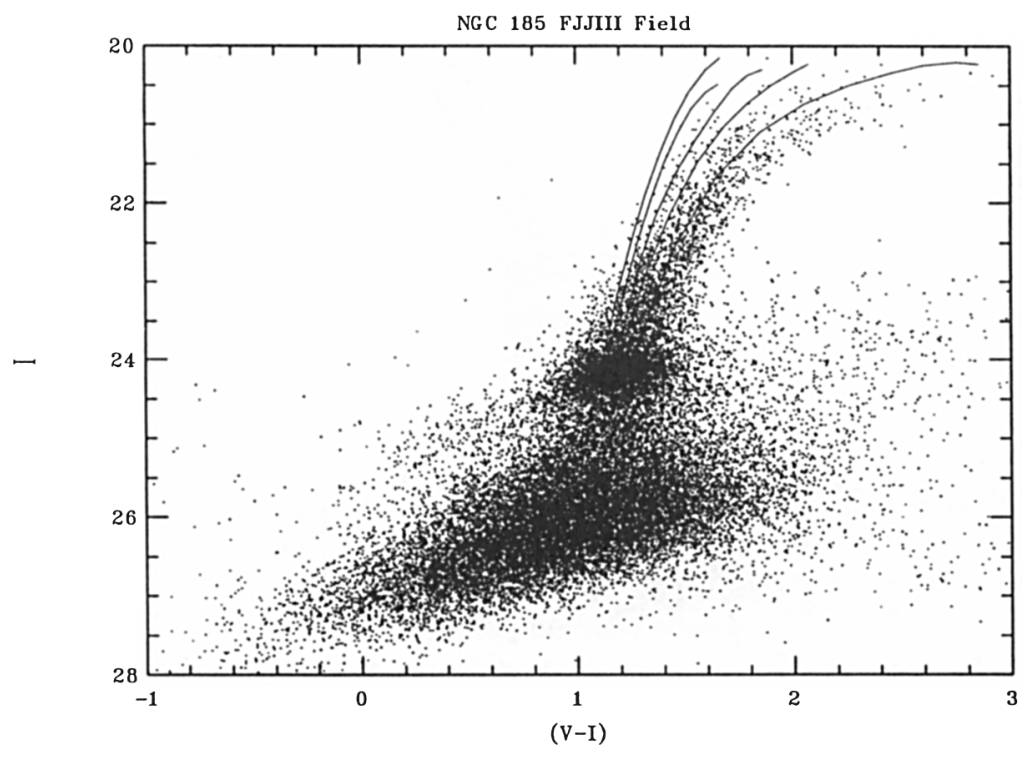

Figure 2. Preliminary $I$ vs. $(V-I)$ CMD for all stars on the PC more than $4.6^{\prime \prime}$ from the center of NGC 185 FJJIII. Curves as in Fig. 1.

and $I \sim 24.5-25.5$, distinctly separated from the red giant branch. The upper giant branch of NGC 185 FJJV follows that of NGC 6752 (the middle curve) quite nicely, indicating a metallicity of $\sim-1.5$. The HB morphology is then about "normal" (similar to that of Galactic GCs of this metallicity). The only previous assessment of the metallicity of this cluster is by Da Costa \& Mould (1988), who derived a value of $-1.8 \pm 0.15$ from an integrated spectrum. Again, our value is preliminary and could certainly change by $\sim 0.3$ dex after the photometry, reddening, distance modulus, etc. are pinned down.

The other clusters that we have so far reduced all show similar CMDs, with metallicities ranging from $\sim-1$ to -2 and HB morphologies in keeping with their metallicities. In the end, we will derive metallicities both from a comparison to the standard giant branches as well as from the slope of the upper giant branch (Sarajedini et al. 1998). We will also assess the HB morphology, derive the magnitude of the HB and investigate the slope of the HB magnitude-metallicity relation.

\section{Preliminary Field Star Results}

Investigations of the field stars in these galaxies are also of great interest. With our deep, high spatial resolution photometry in a variety of regions in these galaxies, we can obtain unprecedented CMDs from which to derive accurate mean metal abundances, their dispersion and the relative proportions of young, intermediate-age, and old stars as a function of galactocentric distance. 


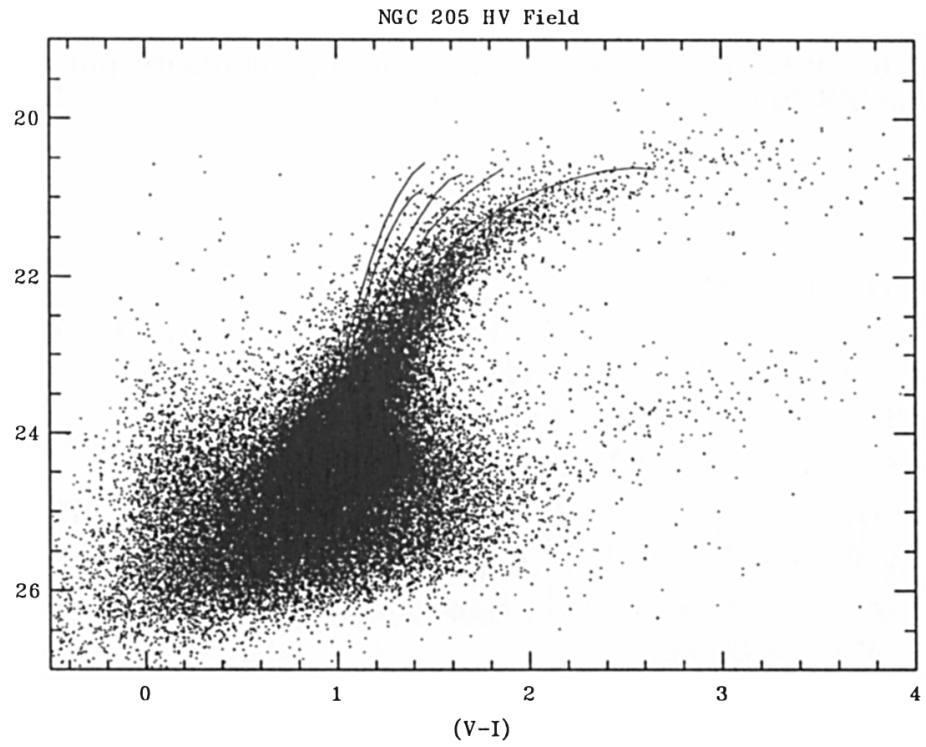

Figure 3. Preliminary $I$ vs. $(V-I)$ CMD for all stars on the PC more than $2.7^{\prime \prime}$ from the center of NGC $205 \mathrm{HV}$. Curves as for Fig. 1, using $E(V-I)=0.045$ and $(m-M)_{V}=24.7$.

Figure 2 shows our CMD for the field stars in the vicinity of NGC 185 FJJIII. This is near (within a few arcminutes) but not at the center of this galaxy. Thus, the blue stars known to inhabit the very center are not present in this field, or only in very small numbers. The CMD shows a strong clump and only a small number of blue HB stars. The upper giant branch indicates a mean metallicity similar to that of 47 Tuc (the reddest standard giant branch, with a metallicity of $\sim-0.75)$, with a large range, from $>-0.5$ to $\sim-1.5$. The distance modulus we will derive from the tip of the giant branch will clearly be very close to that we assumed when fitting the standard giant branches. Also note the lack of any upper AGB stars, indicating any intermediate age population must be small. The metallicity we are finding is higher than derived in previous groundbased studies (Lee et al. 1993, Martinez-Delgado \& Aparicio 1998), which find mean values of $\sim-1.3$. A more distant field shows a very similar CMD, with a very similar mean metallicity and spread, and an apparently more pronounced blue $\mathrm{HB}$, the statistical validity of which remains to be established. Thus, there are apparently no large radial variations in either the mean metallicity or the metallicity range.

Finally, Fig. 3 portrays our CMD of the field stars in the vicinity of NGC 205 HV. This is very close to the center of this galaxy. Thus, the blue stars known to inhabit the very center are seen. The CMD shows a very strong clump and it is impossible to distinguish any blue HB stars from the blue MS stars. The upper giant branch indicates a mean metallicity even higher than that of 47 Tuc, probably at least as high as $\sim-0.5$, ranging up to very high values and down to 
at least -1.5 . There appear to be a few upper AGB stars. A more distant field shows a very similar CMD, with a very similar mean metallicity and spread, but few if any blue MS stars.

\section{References}

Baade, W. 1944, ApJ, 100, 147

Battistini, P., Bonoli, F., Braccesi, A., Federici, L., Fusi Pecci, F., Marano, B., Borngen, F. 1987, A\&AS, 67, 447

Da Costa, G.S., Mould, J.R. 1988, ApJ, 334, 159

Da Costa, G.S., Armandroff, T.E. 1990, AJ, 100, 162

Durrell, P., Harris, W.E., Geisler, D., Pudritz, R. 1996, AJ, 112, 972

Ford, H.C., Jacoby, G., Jenner, D.C. 1977, ApJ, 213, 18

Geisler, D., Sarajedini, A., Lee, M.G., Harris, W.E. 1999, in preparation

Hodge, P.W. 1974, PASP, 86, 289

Hubble, E. 1932, ApJ, 76, 44

Lee, M.G., Freedman, W.L., Madore, B.F. 1993, AJ, 106, 964

Martinez-Delgado, D., Aparicio, A. 1998, AJ, 115, 1462

Miller, B.W., Lotz, J.M., Ferguson, H.C., Stiavelli, M., Whitmore, B.C. 1999, ApJ, in press

Sarajedini, A., Geisler, D., Harding, P., Schommer, R.A. 1998, ApJ, 508, 37

Sargent, W.L.W., Kowal, C., Hartwick, F.D.A., van den Bergh, S. 1977, AJ, 82, 947

Stetson, P.B. 1994, PASP, 106, 250

van den Bergh, S. 1969, ApJS, 19, 145

\section{Discussion}

Hesser: Your metallicity estimates seem to be systematically higher than earlier work. Have you had a chance to reconcile the estimates?

Geisler: Our results are very preliminary as yet, e.g., we have not yet applied aperture corrections. The (fairly small) apparent differences with the Da Costa \& Mould (1988) metallicities may or may not go away when our analysis is complete.

Aparicio: Your data are wonderful!! (1) About NGC 185 (field), Baade reported the discovery of a few blue stars near the center of the galaxy, but your CM diagram seems to have no traces of those stars. What is the reason? (2) Your CM diagram of U 205 shows a well-populated blue-plume. It would correspond to a population 1 Gyr old or younger.

Geisler: (1) The blue stars are very centrally concentrated and our closest field to the center does NOT include the center. (2) Yes. 